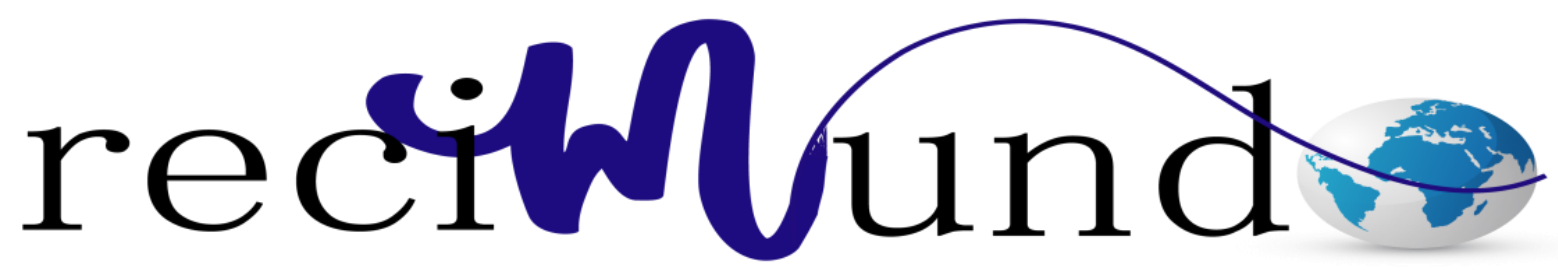

Revista Científica Mundo de la Investigación y el Conocimiento

Cesar Bravo Bermeo ${ }^{\mathrm{a}}$; Carmen Lazo Caicedo ${ }^{\mathrm{b}}$; Sonia Cedeño Loor ${ }^{\mathrm{c}}$; Eduardo Vera Muthre $^{\mathrm{d}}$

Calidad de Atención Primaria de Salud desde la perspectiva de los usuarios. Guayaquil año 2015

Quality of Primary Health Care from the perspective of users.

Guayaquil year 2015

Revista Científica Mundo de la Investigación y el Conocimiento. Vol. 2 núm.3, julio, ISSN: 2588-073X, 2018, pp. 57-72

DOI: 10.26820/recimundo/2.(3).julio.2018.57-72

Editorial Saberes del Conocimiento

Recibido: 05/04/2018

Aceptado: 08/05/2018

Publicado: 30/07/2018

Correspondencia: cesar.bravob@ug.edu.ec
a. Docente Carrera de Medicina. Universidad de Guayaquil; cesar.bravob@ug.edu.ec
b. Docente Carrera de Medicina. Universidad de Guayaquil; carmen.lazoc@ug.edu.ec
c. Docente Carrera de Medicina. Universidad de Guayaquil; leylla.cedenol@ug.edu.ec
d. Docente Carrera de Medicina. Universidad de Guayaquil; eduardo.veram@ug.edu.ec 
Calidad de Atención Primaria de Salud desde la perspectiva de los usuarios. Guayaquil. Año 2015

Vol. 2, núm. 3., (2018)

Cesar Bravo Bermeo; Carmen Lazo Caicedo; Sonia Cedeño Loor; Eduardo Vera Muthre

\section{RESUMEN}

Introducción: sin calidad la Atención Primaria de Salud (APS) caería en descredito, perdería vigencia, se desestabilizaría el sistema y se afectaría la salud de los ecuatorianos más pobres, lo que justifica su evaluación con un método que tenga como actores principales a los usuarios. Objetivo: calificar la calidad del servicio que reciben los usuarios mediante el grado de satisfacción y aclarar la manifiesta y persistente opinión en algunas comunidades contrarias a voces autorizadas del sistema de salud que resaltan la calidad y la calidez de los servicios que ofertan. Material y Método: se trata de un estudio observacional, exploratorio, retrospectivo con enfoque cualitativo-cuantitativo, cuyas unidades de análisis fueron usuarios y centros de APS de la ciudad de Guayaquil, año 2015. Los instrumentos fueron la aplicación de cuestionario a los usuarios y entrevista a los directores de unidades; las dimensiones se dividieron en tangibles e intangibles; la métrica, escala de satisfacción. Resultados: (70.8\%) centros de APS tienen buena y muy buena calificación; 29,2\% regular y mala calificación. Conclusión: si bien la controversia no termina, las cifras demuestran que no se puede generalizar la calidad de atención como mala o buena. La investigación identifico los centros con mayor necesidad de intervención, su potencial de mejora y los distritos mejor calificados en el acciona sanitario.

Palabras claves: Atención primaria, calidad, satisfacción, usuarios. 


\title{
Calidad de Atención Primaria de Salud desde la perspectiva de los usuarios.
}

Guayaquil. Año 2015

Vol. 2, núm. 3., (2018)

Cesar Bravo Bermeo; Carmen Lazo Caicedo; Sonia Cedeño Loor; Eduardo Vera Muthre

\begin{abstract}
Introduction: without the quality of Primary Health Care (PHC), the fall in the discredit, lose the validity, destabilize the system and affect the health of the poorest Ecuadorians, which justifies its evaluation with a method that has as main actors to users. Objective: to qualify the quality of the service that the users receive in the degree of satisfaction and to clarify the manifestation and persistent opinion in some communities opposed to authorized voices of the health system that highlight the quality and the warmness of the services that they offer. Material and methods: This is an observational, exploratory, retrospective study with qualitative-quantitative approach, whose units of analysis were users and PHC centers in the city of Guayaquil, in 2015. The instruments were the object of a questionnaire to users and Interview with unit directors; the dimensions were divided into tangibles and intangibles; the metric, scale of satisfaction. Results: (70.8\%) APS centers have good and very good qualifications; $29.2 \%$ regular and poor rating. Conclusion: although the controversy does not end, the figures show that the quality of care cannot be generalized as bad or good. The research identifies the centers with the mayor's need for intervention, their potential for improvement and the best qualified districts in health care.
\end{abstract}

Keywords: Primary care, quality, satisfaction, users. 


\section{Calidad de Atención Primaria de Salud desde la perspectiva de los usuarios. Guayaquil. Año 2015}

Vol. 2, núm. 3., (2018)

Cesar Bravo Bermeo; Carmen Lazo Caicedo; Sonia Cedeño Loor; Eduardo Vera Muthre

\section{Introducción.}

La salud es un bien individual y colectivo con paradigmas cambiantes a través de los siglos desde, lo mítico-religioso hasta el gran desarrollo científico-técnico que trajo aparejado modelos de atención diferentes. Se pasó del individualizado caritativo al mercantil médicohospitalario que se tradujo en inequidad con mayorías pobres desatendidas. La Declaración de Alma Ata (1978) y la APS fue la respuesta a la injusticia social cuya aplicación con enfoque holístico resolvería el $80 \%$ de los problemas de salud. ${ }^{1,2}$ El Ministerio de Salud Pública (MSP) apunta a que la Atención Primaria de Salud (APS) con calidad sea núcleo del desarrollo de la comunidad, recupere la medicina familiar, descongestione los hospitales y amplíe la cobertura a menor costo. Sin calidad, la APS caería en descredito, perdería vigencia, se desestabilizaría el sistema y se afectaría la salud de los ecuatorianos más pobres, lo que justifica su evaluación con un método que tenga como actores principales a los usuarios ${ }^{3,4}$.

La información de los usuarios que se atienden en el (MSP), obtenida a través de los noticieros y diarios del país, contrasta con la emitida por los responsables de la salud del gobierno actual. Los primeros, específicamente, los que asisten a las unidades de (APS) expresan insatisfacción con la atención que reciben; los segundos, resaltan los avances en las obras físicas, dotación de materiales y recursos humanos; enfatizan sobre el derecho a la salud de los ciudadanos y señalan que la atención es de calidad con calidez. Pese a la notable inversión en salud pública, la percepción y calificación de la calidad del servicio por los usuarios que se atienden en Guayaquil ha sido una constante de quejas y frustraciones que la configuran como mala, hecha pública por los medios de información colectiva. Frente a esta controversia de 


\section{Calidad de Atención Primaria de Salud desde la perspectiva de los usuarios.}

Guayaquil. Año 2015

Vol. 2, núm. 3., (2018)

Cesar Bravo Bermeo; Carmen Lazo Caicedo; Sonia Cedeño Loor; Eduardo Vera Muthre sectores, se plantea esta investigación que tiene como objetivo calificar el nivel de calidad de atención con el grado de satisfacción de los usuarios en los centros de APS de Guayaquil y, de esta forma, aclarar la controversia existente, identificar los centros que requieren mayor intervención, su potencial de mejora y los distritos que mejor califican en los servicios que proporcionan a las comunidades.

La satisfacción puede ser medida desde la visión de las autoridades, los profesionales y los usuarios que, responde a metodologías, dimensiones e indicadores diferentes, pero, independiente del sector investigado, sus resultados son útiles en la toma de decisiones pro mejora de los servicios que se ofertan. ${ }^{5,6,7}$ Sin embargo, existe más evidencia en la literatura médica del determinante significado de la satisfacción de los usuarios en el componente calidad del servicio en el sistema sanitario ${ }^{8,9,10}$ El conocimiento de la información recibida en forma directa de los usuarios resultaría tanto o más importante que la obtenida de los niveles jerárquicos del sistema por lo que, en concordancia con esta realidad se realiza esta investigación tomando como unidades de análisis a los centros de APS del MSP y como fuente primaria de información a los usuarios.

\section{Material y Método.}

El método utilizado es resultado de revisión, modificación y adaptación de estudios previos sobre calidad/satisfacción en APS, de ellos, fueron tomados como referentes principales: a) Aplicación of Patient-Doctor Relationship Questionnaire versión de 9 preguntas aplicadas (PDRQ-9) aplicado a 451 pacientes aleatoriamente seleccionados de una consulta de familia. ${ }^{11,12}$; 


\section{Calidad de Atención Primaria de Salud desde la perspectiva de los usuarios.}

Guayaquil. Año 2015

Vol. 2, núm. 3., (2018)

Cesar Bravo Bermeo; Carmen Lazo Caicedo; Sonia Cedeño Loor; Eduardo Vera Muthre

b) Observación y posterior entrevista telefónica a 152 pacientes que evalúa la información aportada por el médico, la solicitada por el paciente y su participación en la toma de decisiones ${ }^{13}$.

Esta investigación sobre satisfacción/calidad de atención del usuario de los centros de APS de la ciudad de Guayaquil es descriptiva, observacional, prospectiva, con enfoque cuantitativo y cualitativo, realizada en los meses de mayo a octubre del 2015 con una encuesta a los usuarios y/o familiares y una entrevista a directores de centros. Las preguntas relacionadas con tiempo de espera, confort, organización del servicio, afabilidad y cortesía del personal administrativo y la actitud enfermera; la relación profesional-paciente, con el tiempo de consulta, disponibilidad para escuchar, atención a sus dudas y temores, empleo de lenguaje comprensivo y actitud compasiva; con la evolución de las enfermedades, seguimiento de los pacientes y acceso a medicamentos. Evalúa globalmente la atención y, si es recomendable o no el centro de APS.

El universo de APS en la ciudad de Guayaquil lo constituye 86 unidades distribuidas en 9 distritos que integran la zona 8 , ubicados en sectores marginales y medios de la ciudad, todos urbanos. De este total se analizaron 48 unidades de 8 distritos, el distrito 9 con 3 unidades de atención no se incorporó a la investigación por problemas administrativo y de logística (distancia y autorización). Se realizaron encuestas a una muestra de 1057 usuarios y/o familiares y entrevistas a 40 directores de unidades en días y horarios diferentes, previa información y consentimiento. Los instrumentos a utilizar formularios y guías semiestructuradas con preguntas cerradas y abiertas sobre variables tangibles y no tangibles que dicen de la satisfacción y la correspondiente calidad. El formulario de encuesta a los usuarios elaborados sobre la base de tres (3) momentos de atención: pre consulta, consulta, pos consulta. 


\section{Calidad de Atención Primaria de Salud desde la perspectiva de los usuarios.}

Guayaquil. Año 2015

Vol. 2, núm. 3., (2018)

Cesar Bravo Bermeo; Carmen Lazo Caicedo; Sonia Cedeño Loor; Eduardo Vera Muthre

Las unidades de análisis, los centros de APS del sistema de salud ecuatoriano son de 3 categorías: Tipo A, B y C, los primeros, de menor dimensión no tienen especialistas y brindan servicio de medicina general, obstetricia, odontología, son de atención diurna,8 horas; los segundos, más amplios, tienen los mismos servicios que el primero y algunos cuentan con especialidades, pediatría y salud mental, además, toma de muestras para examen, ecografías, pocos atienden 12 horas; los últimos, son prácticamente hospitales básicos, con especialidades, laboratorio, Rx, ecografía, emergencia 24 horas, partos y promoción de la salud.

Los usuarios participantes se incluyeron de forma aleatoria, con el requisito de ser mayores de 18 años, tener instrucción básica con independencia del sexo y de si se trataba de la primera consulta o consultas habituales. En el caso de usuarios que por razones de enfermedad, instrucción o negativa a responder el cuestionario, este fue dirigido al familiar acompañante que cumpliera con las características de inclusión. Los encuestadores, estudiantes de medicina de la Facultad de CC.MM de la Universidad de Guayaquil.

La entrevista a los directores tuvo como propósito comparar las afirmaciones de los usuarios con las del director en lo atinente a recursos y provisión de servicios; contrastar las principales quejas de los usuarios con las recibidas y aceptadas por el director; conocer las causas que, de acuerdo a su opinión, explican las dificultades que motivan las quejas de los usuarios; obtener del director los aspectos con potencial de mejora y datos estadísticos de la unidad a su cargo.

Se obtuvo de cada centro de salud una calificación por separado y sumatoria de las subvariables tangibles (estructura y recursos: humano, material, medicinas) y no tangibles: 
Calidad de Atención Primaria de Salud desde la perspectiva de los usuarios. Guayaquil. Año 2015

Vol. 2, núm. 3., (2018)

Cesar Bravo Bermeo; Carmen Lazo Caicedo; Sonia Cedeño Loor; Eduardo Vera Muthre

tiempo de espera, confort, trato (administrativo, profesional médico y enfermera). A las 9

dimensiones, 4 tangibles y 5 intangibles, se les dio una calificación cualitativa ordinal: malo, regular, bueno, muy bueno, interpretados como insatisfacción, poca satisfacción, satisfacción, mucha satisfacción (Tabla 1), a los que se les asigno un valor numérico, recogidos en una escala: $(<6.49) ;(>6,5-7,49) ;(7,5-8,49) ;(>8,5)$, respectivamente (Tabla 2). Se realizó sumatoria de los valores de las 9 dimensiones de todas las unidades de cada distrito.

Igual, se sumó el valor cuantitativo individualizado y por separado de las dimensiones, intangibles y tangibles de cada unidad de los que se obtuvo la media aritmética, valor que proporciono la calificación de calidad de la unidad. Así registrados, se procedió al análisis comparativo de las dimensiones para establecer las que determinan la condición de: nada recomendable $(<6.49)$, poco recomendable $(6.5-7.49)$, recomendable $(7.5-8.49)$, muy recomendable (>8.5) y en qué medida había participación aislada del componente tangible o intangible o de ambos a la vez.

Una vez establecida la calificación de cada unidad y su ubicación en el distrito correspondiente se pudo graficar el número de unidades de cada distrito y el grado de recomendación de sus unidades, lo que a su vez establece el potencial de mejora da cada unidad y, consecuentemente, de cada distrito (Tabla 3)

\section{Resultados.}

48 centros de APS visitados; 40 directores entrevistados; 1057 usuarios encuestados

Variables tangibles: estructura, materiales, recursos humanos, medicinas 
Calidad de Atención Primaria de Salud desde la perspectiva de los usuarios. Guayaquil. Año 2015

Vol. 2, núm. 3., (2018)

Cesar Bravo Bermeo; Carmen Lazo Caicedo; Sonia Cedeño Loor; Eduardo Vera Muthre

Variables intangibles: tiempo, confort, trato (administrativo, medico, enfermera)

Tabla 1. Calificación de calidad de las unidades evaluadas de APS. Ciudad de Guayaquil. 2015

NR: Nada Recomendable: PR Poco Recomendable: R: Recomendable; MR: Muy Recomendable

\begin{tabular}{|c|c|c|c|c|}
\hline Variables & Tangibles & PR & 7,43 & PR \\
\hline & & $\mathrm{R}$ & 8,02 & $\mathrm{R}$ \\
\hline Distrito 1 & & $\mathrm{R}$ & 7,66 & $\mathrm{R}$ \\
\hline El Cóndor & 8,1 & PR & 8,16 & $\mathrm{R}$ \\
\hline La Floresta & 7,83 & $\mathrm{R}$ & 7,85 & $\mathrm{R}$ \\
\hline Guasmo Central & 7,4 & & & \\
\hline CS.3 & 8,11 & & & \\
\hline Casitas del Guasmo & 8,08 & & & \\
\hline 25 de Enero & 7,48 & & & \\
\hline Florida & 7,68 & $\mathrm{R}$ & 7,52 & $\mathrm{R}$ \\
\hline Proletarios sin Tierra & 7,87 & $\mathrm{R}$ & 7,59 & $\mathrm{R}$ \\
\hline 8 & & & & \\
\hline Distrito 2 & & & & \\
\hline Rumiñahui & 7,70 & $\mathrm{R}$ & 7,86 & $\mathrm{R}$ \\
\hline Trinitaria 1 & 7,63 & $\mathrm{R}$ & 7,9 & $\mathrm{R}$ \\
\hline Fertisa & 7,33 & PR & 7,74 & $\mathrm{R}$ \\
\hline Trinitaria 2 & 7,61 & $\mathrm{R}$ & 8,73 & MR \\
\hline Guangala & 7,7 & $\mathrm{R}$ & 8,13 & $\mathrm{R}$ \\
\hline 5 & & & & \\
\hline
\end{tabular}


Calidad de Atención Primaria de Salud desde la perspectiva de los usuarios. Guayaquil. Año 2015

Vol. 2, núm. 3., (2018)

Cesar Bravo Bermeo; Carmen Lazo Caicedo; Sonia Cedeño Loor; Eduardo Vera Muthre

\begin{tabular}{|l|}
\hline Distrito 3 \\
\hline SAIS \\
\hline CS.14 \\
\hline Julián Coronel \\
\hline $\begin{array}{l}\text { Lizardo García y } \\
\text { Venezuela }\end{array}$ \\
\hline Cuenca y Noguchi \\
\hline Barrio Lindo \\
\hline Cuenca y la 11 \\
\hline $\begin{array}{l}\text { Machala y Camilo } \\
\text { Destruge }\end{array}$ \\
\hline $\begin{array}{l}\text { Callejón Parra y } \\
\text { Valenzuela }\end{array}$ \\
\hline
\end{tabular}

\begin{tabular}{|c|c|c|c|}
\hline 8,1 & $\mathrm{R}$ & 7,91 & $\mathrm{R}$ \\
\hline 7,83 & $\mathrm{R}$ & 7,78 & $\mathrm{R}$ \\
\hline 7,4 & $\mathrm{PR}$ & 7,5 & $\mathrm{R}$ \\
\hline & $\mathrm{R}$ & 7,89 & $\mathrm{R}$ \\
\hline 8,11 & $\mathrm{R}$ & 8,02 & $\mathrm{R}$ \\
\hline 7,67 & $\mathrm{PR}$ & 7,89 & $\mathrm{R}$ \\
\hline 7,09 & $\mathrm{R}$ & 8,17 & $\mathrm{R}$ \\
\hline 7,69 & $\mathrm{R}$ & 7,87 & $\mathrm{R}$ \\
\hline 7,61 & $\mathrm{R}$ & 8.62 & $\mathrm{MR}$ \\
\hline 7,77 & & & \\
\hline
\end{tabular}

\begin{tabular}{|l|}
\hline Distrito 4 \\
\hline Santiago de Guayaquil \\
\hline San. José \\
\hline Cisne 2 \\
\hline Cs. N8 \\
\hline CS N13 \\
\hline CS N6 \\
\hline 46 y Rosendo Avilés \\
\hline La Colmena \\
\hline
\end{tabular}

\begin{tabular}{|c|c|c|c|}
\hline 7,85 & $\mathrm{R}$ & 7,93 & $\mathrm{R}$ \\
\hline 7,79 & $\mathrm{R}$ & 8,11 & $\mathrm{R}$ \\
\hline 7,97 & $\mathrm{R}$ & 7,95 & $\mathrm{R}$ \\
\hline 7,59 & $\mathrm{R}$ & 7,95 & $\mathrm{R}$ \\
\hline 8,03 & $\mathrm{R}$ & 8,3 & $\mathrm{R}$ \\
\hline 7,77 & $\mathrm{R}$ & 7,85 & $\mathrm{R}$ \\
\hline 7,88 & $\mathrm{R}$ & 7,93 & $\mathrm{R}$ \\
\hline 7,85 & $\mathrm{R}$ & 8,09 & $\mathrm{R}$ \\
\hline & & & \\
& & & \\
\hline & $\mathrm{R}$ & 7,73 & $\mathrm{R}$ \\
\hline 7,65 & $\mathrm{R}$ & 7,94 & $\mathrm{R}$ \\
\hline 8,24 & & & \\
\hline & & & \\
\hline
\end{tabular}


Calidad de Atención Primaria de Salud desde la perspectiva de los usuarios. Guayaquil. Año 2015

Vol. 2, núm. 3., (2018)

Cesar Bravo Bermeo; Carmen Lazo Caicedo; Sonia Cedeño Loor; Eduardo Vera Muthre

\begin{tabular}{|c|c|c|c|c|}
\hline \multicolumn{5}{|l|}{ Distrito 6} \\
\hline Mapasingue Este & 7,48 & PR & 7,97 & $\mathrm{R}$ \\
\hline Mapasingue Oeste & 7,4 & PR & 7,82 & $\mathrm{R}$ \\
\hline Prosperina & 7,38 & PR & 7,61 & $\mathrm{R}$ \\
\hline Estrella de Belén & 7,69 & $\mathrm{R}$ & 7,87 & $\mathrm{R}$ \\
\hline Francisco Jácome & 8,25 & $\mathrm{R}$ & 8,06 & $\mathrm{R}$ \\
\hline Martha Roldos & 7,91 & $\mathrm{R}$ & 7,8 & $\mathrm{R}$ \\
\hline Ferroviaria & 7,8 & $\mathrm{R}$ & 7,21 & $\mathrm{PR}$ \\
\hline Ficoa de Montalvo & 8,28 & $\mathrm{R}$ & 7,5 & $\mathrm{R}$ \\
\hline Luchadores del Norte & 8,06 & $\mathrm{R}$ & 8,07 & $\mathrm{R}$ \\
\hline Gallegos Lara & 7,23 & PR & 7,93 & $\mathrm{R}$ \\
\hline \multicolumn{5}{|l|}{ (2) } \\
\hline \multicolumn{5}{|l|}{ Distrito 7} \\
\hline Orquídeas & 7,41 & PR & 7,88 & $\mathrm{R}$ \\
\hline Pascuales & 7,75 & $\mathrm{R}$ & 8,2 & $\mathrm{R}$ \\
\hline Bastión Popular & 6,75 & $\mathrm{PR}$ & 7,99 & $\mathrm{R}$ \\
\hline Vergeles Norte & 6,48 & NR & 6,61 & $\mathrm{PR}$ \\
\hline \multirow{2}{*}{\multicolumn{5}{|c|}{ Distrito 8}} \\
\hline & & & & \\
\hline Flor de Bastión & 7,86 & $\mathrm{R}$ & 7,67 & $\mathrm{R}$ \\
\hline Monte Sinaí & 7,1 & PR & 7,55 & $\mathrm{R}$ \\
\hline 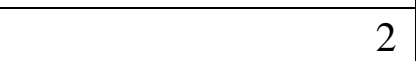 & & & & \\
\hline $\begin{array}{r}\text { Total } \\
48\end{array}$ & & & & \\
\hline
\end{tabular}


Calidad de Atención Primaria de Salud desde la perspectiva de los usuarios. Guayaquil. Año 2015

Vol. 2, núm. 3., (2018)

Cesar Bravo Bermeo; Carmen Lazo Caicedo; Sonia Cedeño Loor; Eduardo Vera Muthre

Tabla 2. Número y porcentaje de recomendación de unidades en función de la evaluación de calidad por los usuarios

De acuerdo con la escala empleada, de 48 centros analizados, NR: 1(2.1\%); PR: 13 (27.1\%);

R: 32 (66.6\%); MR 2 (4,2\%)

Escala

\begin{tabular}{|l|l|}
\hline ESCALA & \\
\hline NR: Nada & \\
Recomendable & $<6.49$ \\
\hline PR: Poco & $>6.5-$ \\
Recomendable & 7.49 \\
\hline R: & $>7.5-$ \\
Recomendable & 8.49 \\
\hline MR: Muy & \\
Recomendable & $>8.5$ \\
\hline
\end{tabular}

\begin{tabular}{|l|c|c|}
\hline & No & $\%$ \\
\hline $\begin{array}{l}\text { Total: nada } \\
\text { recomendable }\end{array}$ & 1 & 2.1 \\
\hline $\begin{array}{l}\text { Total : poco } \\
\text { recomendable }\end{array}$ & 13 & 27.1 \\
\hline $\begin{array}{l}\text { Total: } \\
\text { recomendable }\end{array}$ & 32 & 66.6 \\
\hline $\begin{array}{l}\text { Total : muy } \\
\text { recomendable }\end{array}$ & 2 & 4.2 \\
\hline $\begin{array}{l}\text { Total de } \\
\text { unidades }\end{array}$ & $\mathbf{4 8}$ & 100.0 \\
\hline
\end{tabular}

Tabla 3. Los distritos y el grado de recomendación de sus unidades derivada de la evaluación de calidad

\begin{tabular}{|c|c|c|c|c|c|}
\hline Distrito & $\begin{array}{c}\text { Nada } \\
\text { recomendable }\end{array}$ & $\begin{array}{c}\text { Poco } \\
\text { recomendable }\end{array}$ & Recomendable & $\begin{array}{c}\text { Muy } \\
\text { Recomendable }\end{array}$ & Total \\
\hline D 1 & & $\mathbf{2}$ & $\mathbf{6}$ & $\mathbf{8}$ & $\mathbf{5}$ \\
\hline D 2 & & $\mathbf{1}$ & $\mathbf{3}$ & $\mathbf{1}$ & $\mathbf{9}$ \\
\hline D 3 & & $\mathbf{2}$ & $\mathbf{6}$ & $\mathbf{8}$ \\
\hline D 4 & & & $\mathbf{8}$ & & $\mathbf{2}$ \\
\hline D 5 & & $\mathbf{5}$ & $\mathbf{2}$ & & $\mathbf{1 0}$ \\
\hline D 6 & & $\mathbf{2}$ & $\mathbf{1}$ & & $\mathbf{4}$ \\
\hline D 7 & $\mathbf{1}$ & $\mathbf{1}$ & $\mathbf{1}$ & & $\mathbf{4 8}$ \\
\hline D 8 & & $\mathbf{1 3}$ & $\mathbf{3 2}$ & $\mathbf{2}$ & \\
\hline Total & $\mathbf{1}$ & & & & \\
\hline
\end{tabular}

Tienen mayor número de centros recomendables los distritos $1,2,3,4,5$, del 4 y 5 todos sus centros caen dentro de esta categoría; el distrito 6 tiene por igual número recomendables y poco recomendables; el distrito 7 es el peor categorizado con 1 centro nada recomendable y 2 poco recomendables; finalmente, el distrito 8 comparten por igual 1 unidad recomendable y otra poco recomendable. 


\section{Calidad de Atención Primaria de Salud desde la perspectiva de los usuarios.}

Guayaquil. Año 2015

Vol. 2, núm. 3., (2018)

Cesar Bravo Bermeo; Carmen Lazo Caicedo; Sonia Cedeño Loor; Eduardo Vera Muthre

\section{Discusión.}

La utilidad de la satisfacción del usuario para evaluar la calidad de la atención como predictor, instrumento u opinión, ha convertido a este parámetro en una herramienta de valor creciente tanto para el marketing como en la investigación en servicios sanitarios. ${ }^{14}$ Sin embargo, dado su carácter multidimensional, que complejiza su aplicación, determina que las investigaciones respondan a un determinado número de variables que el investigador selecciona de acuerdo al contexto, propósito y objetivos de la investigación. Bajo este considerando, la investigación pudiera reconocerse como muy amplia o muy reducida, lo fundamental es que la información captada sea una traducción de la realidad, que aborde los componentes de la calidad: recursos, proceso y resultados y, con ellos, se pueda globalizar la opinión del usuario del servicio que recibe.

Reconocida la variable satisfacción como una percepción compleja ${ }^{15}$ que combina aspectos subjetivos de la personalidad con componentes bien definidos de la oferta de servicio, su definición, evaluación y calificación, resulta un esfuerzo de aproximación para el que se han implementado enfoques diversos, estrategias metodológicas múltiples, e instrumentos de aplicación variados con los que no siempre se tendrá la certeza de haber obtenido la expresión más honesta, cierta, acerca del cumplimiento de las expectativas y necesidades del usuario y, consecuentemente, la puntuación que más exactamente califique la calidad.

Con esta incertidumbre la literatura sobre la satisfacción de los usuarios con la calidad en los diferentes niveles de atención de salud, trae cada vez más novedades, particularmente, sobre los métodos empleados para su estudio, cuyo número se amplía en función de la 


\section{Calidad de Atención Primaria de Salud desde la perspectiva de los usuarios. Guayaquil. Año 2015}

Vol. 2, núm. 3., (2018)

Cesar Bravo Bermeo; Carmen Lazo Caicedo; Sonia Cedeño Loor; Eduardo Vera Muthre

consideración de cuál es o cuáles son las dimensiones o variables que mejor interpreten lo subjetivo de la satisfacción y mejor definan lo objetivo de la calidad.

Este estudio plantea un método que procura ser abreviado y práctico por el número y la combinación de las variables seleccionadas, si se compara con estudios del mismo orden.

\section{Conclusión.}

Las preguntas, las calificaciones y los números sirvieron para apreciar que algunos centros de APS del sistema de salud pública ecuatoriano, ubicados en Guayaquil, tienen falencias físicas y humanas que impiden a los usuarios, en su gran mayoría pobres, recibir una atención que les proporcione satisfacción y calificarlas de buena calidad. Por otra parte, un buen sector de la población reconoce de los centros, un grado importante de satisfacción y le concede una calificación que se la interpreta como buena calidad de desempeño sanitario. En cuanto a la controversia entre las comunidades y las autoridades de la salud pública, los datos de la investigación concluyen: no se puede generalizar que la atención del sistema de APS es de buena o de mala calidad, hay bien calificados en mayor número $(70,8 \%)$ y mal calificados en menor número $(29,2 \%)$, estos últimos con mayor potencial de mejora. Así mismo, se identifica los distritos y sus unidades que requieren mayor y pronta intervención, como también el grado de recomendación derivada de la calificación de calidad.

Sería de desear que nuevos estudios sobre el tema en otras ciudades y regiones del país tengan como referencia y punto de partida el enfoque aquí presentado; queda asimismo, el espacio abierto para seguir ahondando y elaborando nuevas propuestas sobre los momentos de atención, sus variables e indicadores. 


\section{Calidad de Atención Primaria de Salud desde la perspectiva de los usuarios.}

Guayaquil. Año 2015

Vol. 2, núm. 3., (2018)

Cesar Bravo Bermeo; Carmen Lazo Caicedo; Sonia Cedeño Loor; Eduardo Vera Muthre

\section{Bibliografía}

1. Starfield B. Equidad en salud y atención primaria: una meta para todos. Rev Ger y Polít de Salud, 2001; 1(1): 7-16

2. Organización Mundial de la Ssalud. Atención Primaria de Salud. Informe de la Conferencia Internacional sobre Atención Primaria de Salud Alma Ata, URSS, Ginebra 6-12 septiembre de 1978

3. Lemus. Aragüés y Oroz, V, Luccioni M. Salud Publica y Atención Primaria de la Salud. 1ed. Ciudad Autónoma de Buenos Aires: Corpus Libros Médicos y Científicos, 2013

4. González Gallardo E. Calidad de la Atención Medica: la diferencia entre la vida o la muerte. Rev Dig Univ. 2012; 13(8).

5. Borre H. Patient satisfaction. And attribute of quality care? Quality Review Bulletin 1978; 13:106-108

6. Paganini JM. Et al. Evaluación de los sistemas de salud y la estrategia de APS. Revista Argentina de Salud Pública. 2010;1(2)

7. Cuba-Fuentes, M, Jurado A, Estrellas E. La atención primaria y el grado de satisfacción de los usuarios de un establecimiento de primer nivel de atención. Rev.Med Hered 2011; 22:4-9.

8. Guzmán, J. Vargas-Hernández. Modelo "Plural Integral" de atención primaria a la salud y satisfacción de los usuarios. Criterio Libre 2013; 11(18): 155-168

9. Acosta, L. et al. Análisis de satisfacción del usuario en centros de salud del primer nivel de atención en la provincia de Córdova. Argentina. Enfermería Global. Revista electrónica trimestral de Enfermería. 2011; 21.

10. Jiménez-De Gracia. et al. Opiniones de los médicos de familia acerca de la implicación de los pacientes en la toma de decisiones, un estudio con grupos focales. Atención Primaria. 2012; 44(7).

11. Martin-Fernández, J. et al. Satisfacción del paciente con la relación con su médico de familia: un estudio con el Patient-Doctor Relationship Questionnaire. Atención Primaria.2010; 42(4):196-205

12. Mingote, J.C. et al. Psychometric validation of the Spanish version of the PatientDoctor Relationship Questionnaire (PDRQ). Actas Esp Psiquiatr 2009; 37(2): 94-100

13. Alleman, I. Kunz, R, Blozik, E. Instruments to assess patient satisfaction after teleconsultation and triage: a systematic review. Patient Prefer Adherence. 2104; 8: 893-907

14. Mira JJ, Aranaz J. La satisfacción del paciente como una medida del resultado de la atención sanitaria. Med Clin (Barc) 2000:114 (3):26-33 
Calidad de Atención Primaria de Salud desde la perspectiva de los usuarios. Guayaquil. Año 2015

Vol. 2, núm. 3., (2018)

Cesar Bravo Bermeo; Carmen Lazo Caicedo; Sonia Cedeño Loor; Eduardo Vera Muthre

15. Papp, R y cols. Perceptions of quality in primary health care: perspectives of patients and professionals based on focus group discussions. BMC Family Practice 2014; $15: 128$. 